\title{
FULLY CONVOLUTIONAL SPATIO-TEMPORAL MODELS FOR REPRESENTATION LEARNING IN PLASMA SCIENCE
}

\author{
Ge Dong, ${ }^{1, *}$ Kyle Gerard Felker, ${ }^{2}$ Alexey Svyatkovskiy, ${ }^{3}$ \\ William Tang, ${ }^{1, \dagger} \mathcal{E}$ Julian Kates-Harbeck ${ }^{1, \dagger}$ \\ ${ }^{1}$ Princeton Plasma Physics Laboratory, Princeton, New Jersey, 08540, USA \\ ${ }^{2}$ Argonne National Laboratory, Lemont, Illinois, 60439, USA \\ ${ }^{3}$ Microsoft Corporation, Redmond, Washington, 98052, USA \\ *Address all correspondence to: Ge Dong, Princeton Plasma Physics Laboratory, \\ Princeton, New Jersey, 08540, USA, E-mail: gdong@princeton.edu \\ ${ }^{\dagger}$ William Tang and Julian Kates-Harbeck supervised the work jointly.
}

Original Manuscript Submitted: 11/10/2020; Final Draft Received: 1/29/2021

We have trained a fully convolutional spatio-temporal model for fast and accurate representation learning in the challenging exemplar application area of fusion energy plasma science. The onset of major disruptions is a critically important fusion energy science issue that must be resolved for advanced tokamak plasmas such as the $\$ 25 B$ burning plasma international thermonuclear experimental reactor (ITER) experiment. While a variety of statistical methods have been used to address the problem of tokamak disruption prediction and control, recent approaches based on deep learning have proven particularly compelling. In the present paper, we introduce further improvements to the fusion recurrent neural network (FRNN) software suite, which delivered cross-machine disruption predictions with unprecedented accuracy using a large database of experimental signals from two major tokamaks. Up to now, FRNN was based on the long short-term memory (LSTM) variant of recurrent neural networks to leverage the temporal information in the data. Here, we implement and apply the "temporal convolutional neural network (TCN)" architecture to the time-dependent input signals. This allows highly optimized convolution operations to carry the majority of the computational load of training, thus enabling a reduction in training time, and the effective use of highperformance computing resources for hyperparameter tuning. At the same time, the TCN-based architecture achieves better predictive performance when compared with the LSTM architecture for various tasks for a representative fusion database.

\section{INTRODUCTION}

Deep learning has become an increasingly important methodology for the effective analysis and interpretation of big data in modern social and scientific areas (Webb, 2018; Schmidt et al., 2019; Reichstein et al., 2019). In this study, we discuss the application of deep-learning models in the prominent exemplar problem of disruption predictions in tokamaks (Kates-Harbeck et al., 2019), 
which are magnetic fusion experimental devices with large numbers of advanced diagnostics to monitor spatio-temporal plasma performance.

In many toroidal plasma devices such as tokamaks and spherical toruses (STs) (Gerhardt et al., 2013), disruptions are observed as sudden and dangerous events that induce rapid release of particles and energy to the device wall (Schuller, 1995). A typical disruption brings the plasma experiment (the "shot") to an abrupt end and, because of the associated large rapid energy release, it can also seriously damage the device-especially in larger systems such as ITER (Strait et al., 2019). Accordingly, the development of a plasma control system (PCS) with the ability to reliably detect and subsequently mitigate or avoid the majority of the disruption events (Hollmann et al., 2015) is broadly regarded as the most important milestone for establishing the viability of future larger tokamak devices to deliver a fusion energy reactor.

To robustly mitigate or prevent disruptions, the first step for the PCS is to accurately predict disruptions as early as possible. Traditional methods for disruption studies and predictions range from using simple empirical formulae and analytic expressions to more sophisticated firstprinciples-based simulations, such as the magnetohydrodynamic (MHD) models (Glasser and Kolemen, 2018) and the gyrokinetic models (Liu et al., 2014). These simulations are used to study the dynamics and mechanisms of disruptions, and accordingly to advance their prediction and also their possible avoidance through active plasma control. Empirical expressions and even MHD models are generally simple and fast enough to be implemented in the PCS and can thereby aid real-time disruption predictions and control. However, these models often contain insufficient physics information to correctly predict complex or novel scenarios, including device operations for different parametric and/or hardware regimes, such as those involving, for example, reactor-relevant wall materials (Matthews et al., 2011). In order to better capture nonlinear dynamics and physics associated with realistic magnetic geometry, large-scale first-principlesbased simulations can be engaged. However, the vast computational resources required to allow real-time predictive capabilities for such simulations make an implementation in the PCS infeasible. Consequently, complementary to the two aforementioned categories of models, emerging big-data-driven methodologies have become an increasingly powerful modern approach addressing the grand challenge of prediction and control of tokamak disruptions (Pautasso et al., 2002). We have accordingly developed a deep-learning capability of general computational science interest that is capable of enabling significant progress towards resolving this major application science exemplar problem via effective utilization of the hardware capabilities of modern leadership class supercomputing systems. This involves the training of multiple models with distinct architectures within a single software suite adaptable to different temporal and spatial learning tasks - with natural connections to enabling ensemble schemes for highly accurate prediction.

We focus on and refer to models that can be easily built and extended in a layered fashionas well as optimized (trained) using automatic differentiation and back-propagation (such as the neural networks) - as "deep-learning" models. Machine-learning models that do not have this property are referred to as "classical" models. Examples of classical models include support vector machines (López et al., 2012) and random forest algorithms (Rea et al., 2019), both of which have been applied successfully to advance disruption prediction capability. So far, a key advantage of deep-learning models has been their ability to perform cross-machine predictionsforecasting the plasma behavior in an experiment never seen during training and or validation. This is especially key for establishing the relevance of such studies for ITER, as ITER will not be able to withstand enough disruptions for a large training dataset (de Vries et al., 2016).

In this paper, we introduce the implementation of a temporal convolutional neural network (TCN) (Oord et al., 2016) for the temporal representation of the extensive database of fusion 
energy science (FES) input signals of interest (Kates-Harbeck et al., 2019). The associated TCN architecture based on dilated causal convolutions (Bai et al., 2018) has some advantages compared with the long short-term memory (LSTM) architecture in FRNN. The TCN architecture will be introduced in detail in the next section.

Comparisons between TCN and LSTM-based architectures for various fusion databases are described in detail in Section 3 of this paper. While TCN-based architectures generally achieve superior predictive power and improved computational performance, we note that for certain tasks and databases, the original LSTM-based model can still outperform all alternatives. From a general computational science perspective, this finding highlights the importance of maintaining multiple architectures in a modern software suite such as FRNN. Moreover, the choice of temporal processing layer (such as LSTM cell versus TCN) can be viewed as a high-level architectural hyperparameter for FRNN. This framing aligns with our vision for an AI/DL-based platform with flexible and adaptive model architectures that can be automatically hyperparameter-tuned for various tasks and databases associated, for example, with future fusion plasma predictions and analysis tasks. This in turn has implications for building capabilities to face future real-time plasma control challenges. To increase the robustness of our models, we developed a noise-aware training scheme within the FRNN framework. The noise-aware training can help achieve predictive capabilities of the deep-learning-based models during real-time experiment in scenarios where certain diagnostics are unavailable or random data contamination happens.

\section{MODEL ARCHITECTURE}

Figure 1 shows the schematic of the new deep-learning architecture based on TCNs introduced here into FRNN. As in the previous LSTM-based models, the TCN-based FRNN models process inputs composed of two main types of signals: 0D scalar signals (such as the plasma current), and $1 \mathrm{D}$ profile signals (such as the electron temperature profile). The descriptions and numerical properties of the $14 \mathrm{OD}$ and 2 1D signals can be found in Table 1. Example time series of signals available on DIII-D will be shown along with the prediction results in the next section. At each time step, the 1D profiles are first "spatially" processed by a sub-network consisting of $\mathrm{N}_{s}$ convolutional layers. The output of this network contains a representation of the 1D features, as shown by the blue bar in Fig. 1. The 1D features are then concatenated with the 0D scalar signals, and together form the complete input channels for the $\mathrm{N}_{t}$ dilated convolutional layer blocks. Each of these convolutional layer blocks consists of dilated causal convolutional layers, activation layers, optional dropout layers, normalization layers, as well as an additive identity map (which maintains the stability of the neural network when it becomes "deep"). Details of the convolutional layer blocks were introduced in Bai et al. (2018).

Compared with the LSTM-based models, TCN-based models have two main advantages for processing the temporal information. First, instead of carrying over historical information in a recurrent fashion, the TCN directly fetches it through a time series, and can accordingly "remember" such information from a more distant past. How to effectively learn long-term dependencies for predictions involving time series databases is an active area of modern computational science research. While the LSTM is an improved architecture over the standard recurrent neural network (RNN) - which has short memory due to the exploding and vanishing gradient problems (Bengio et al., 1994) - it can still lose distant information through operations on the cell state, which carries its long term memory. While the temporal receptive field of regular convolutions grows linearly with network depth (necessitating prohibitively deep architectures for long-term memory tasks), the temporal receptive field of dilated convolutions grows

Volume 2, Issue 1, 2021 


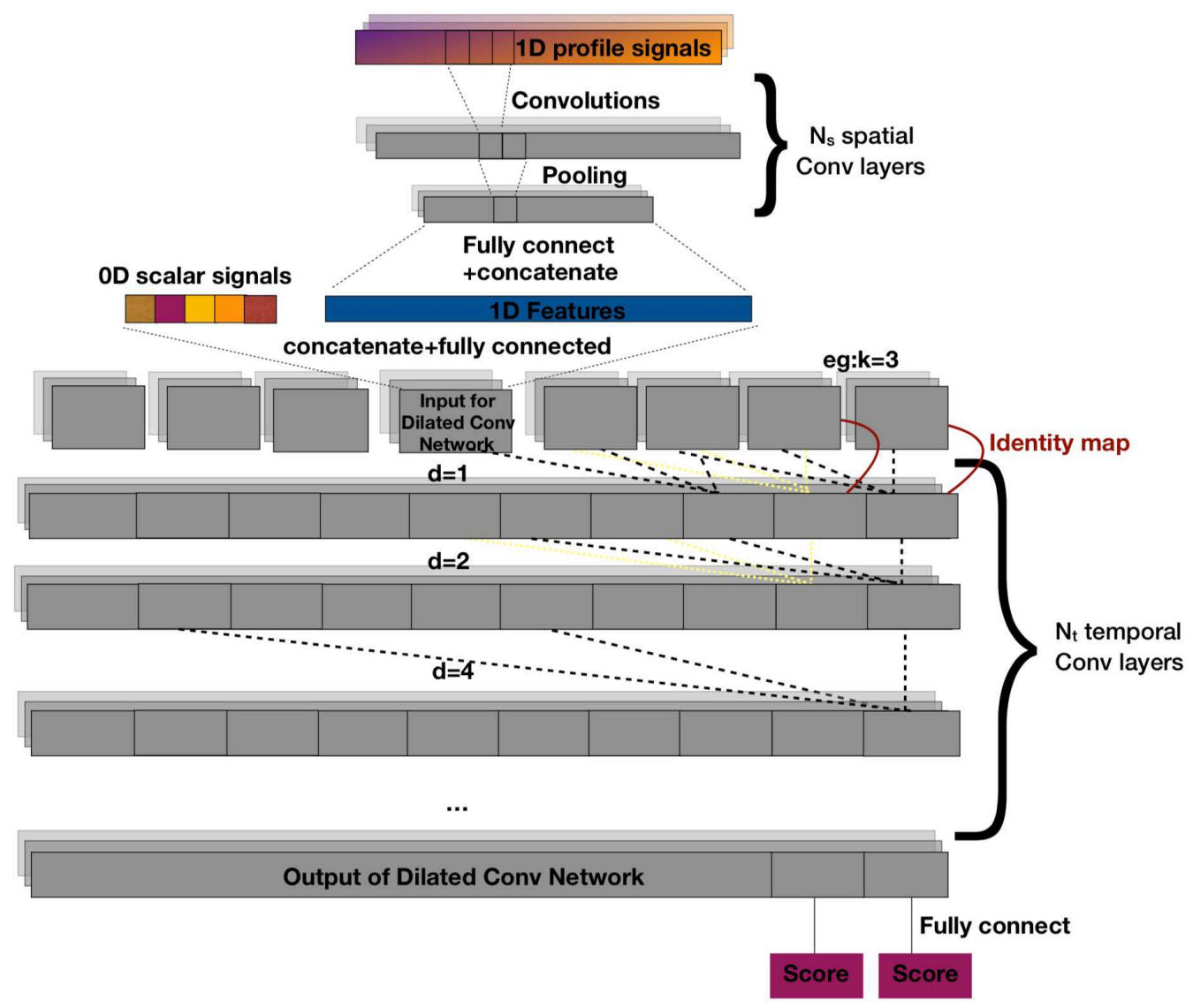

FIG. 1: The detailed schematic of our deep-learning model based on temporal convolutional architecture. $\mathrm{N}_{s}$ is the number of convolutional layers for spatial information processing at each time step, $\mathrm{N}_{t}$ is the number of dilated temporal convolutional layers, $\mathrm{d}$ is the dilation factor, and $\mathrm{k}$ is the filter size for the dilated convolutional layers.

exponentially with network depth. TCNs can thus capture long distance dependencies with a modest number of layers.

The second advantage of the TCN architecture is that it is easily amenable to accelerated training via model parallelism. The serial implementation of the model is straightforward; i.e., in addition to fully connected layers and activation functions, only the convolution operation (applied to both the spatial and temporal representations) is required. The resulting feedforward network does not incorporate gated functions or recurrent connections. In contrast, the recurrent nature of the LSTM and other models based on RNN are generally hard to parallelize within training examples on the model level, as commented by Bai et al. (2018). We highlight the fact that for our exemplar FES application of interest, the training and inference on long experimental runs with dense temporal measurements leads to a large memory footprint-thereby making the TCN's efficient model parallelism a particularly attractive feature in the context of highperformance computing (HPC). Specifically, the TCN architecture can effectively utilize the hardware capabilities of modern leadership class supercomputing systems-a fact that will be demonstrated later in this paper. 
TABLE 1: Measured DIII-D and JET experimental signals used in FRNN

\begin{tabular}{|c|c|c|}
\hline Signal description & Numerical scale DIII-D & Numerical scale jet \\
\hline Plasma current & $3.8 \mathrm{E}-1 \mathrm{MA}$ & $5.03 \mathrm{E}-1 \mathrm{MA}$ \\
Plasma current target & $3.9 \mathrm{E}-1 \mathrm{MA}$ & Not available on JET \\
Plasma current error & $3.1 \mathrm{E}-2 \mathrm{MA}$ & Not available on JET \\
Plasma current direction & 1.0 & Not available on JET \\
Internal inductance & $2.02 \mathrm{E}-1$ & $1.51 \mathrm{E}-1$ \\
Plasma density & $1.19 \mathrm{E}+19 \mathrm{~m}^{-3}$ & $4.69 \mathrm{E}+19 \mathrm{~m}^{-3}$ \\
Input power (beam for DIII-D) & $1.85 \mathrm{E}+6 \mathrm{~W}$ & $4.47 \mathrm{E}+6 \mathrm{~W}$ \\
Radiated power core & $4.58 \mathrm{E}+2 \mathrm{~W}$ & $4.05 \mathrm{E}+4 \mathrm{~W}$ \\
Radiated power edge & $4.94 \mathrm{E}+2 \mathrm{~W}$ & $2.72 \mathrm{E}+4 \mathrm{~W}$ \\
Stored energy & $2.79 \mathrm{E}+5 \mathrm{~J}$ & $1.2 \mathrm{E}+6 \mathrm{~J}$ \\
Locked-mode amplitude & $1.14 \mathrm{E}-6 \mathrm{~T}$ & $5.72 \mathrm{E}-5 \mathrm{~T}$ \\
Safety factor Q95 & 1.0 & 1.0 \\
Normalized plasma pressure & $6.91 \mathrm{E}-3$ & Not available on JET \\
Input beam torque & $1.47 \mathrm{Nm}$ & Not available on JET \\
Electron temperature profile & $9.53 \mathrm{E}-1 \mathrm{keV}^{-3}$ & $1.53 \mathrm{keV}$ \\
Electron density profile & $1.47 \mathrm{E}+19 \mathrm{~m}^{-3}$ & $2.98 \mathrm{E}+19 \mathrm{~m}^{-3}$ \\
\hline
\end{tabular}

With a chosen time step of $1 \mathrm{~ms}$, and a typical shot duration on DIII-D of several seconds (tens of seconds on Joint European Torus, JET), the typical length of a time series in our dataset is thousands to tens of thousands of steps. Since the effective history length of a normal convolutional layer is $\mathrm{k}-1$, where $\mathrm{k}$ is the convolution filter size, regular convolutional neural networks have a receptive field linear in depth. By contrast, that of dilated convolutional neural networks, where each layer has effective history length of $(\mathrm{k}-1) * \mathrm{~d}$, can be exponential if the dilation factor $\mathrm{d}$ is grown accordingly. The need for dilations (see dashed connections in Fig. 1) arises if we need to allow the network output near the end of the shot to depend on early plasma behaviorand to be able to do so without requiring unwieldy network depth.

The output of the dilated convolutional layer blocks feeds into a final fully connected layer that combines the information from all of the hidden units. It then outputs the disruption score which measures the likelihood of an imminent disruption at each time step. The definition of this disruption score, or the "target" that FRNN is trained on, is effectively a hyperparameter that can be tuned. A detailed explanation of the tradeoffs involved in the selection of the target function is provided in the "Methods" section of Kates-Harbeck et al. (2019).

For a disruptive shot, if the disruption score rises above the preset "alarm threshold" before the "warning time" (the latest acceptable alarm time before the actual disruption), it would count as a true-positive prediction. For a nondisruptive shot, if the disruption score rises above the alarm threshold at any time, it would count as a false-positive prediction. By shifting the alarm threshold from minus infinity (model predicts disruption for every shot) to infinity (model predicts no disruption for any shot), a receiver operating characteristic curve (ROC curve) is produced. We use the area under the curve (AUC) of the ROC curve on the test data prediction results as the metric to evaluate model performance. Although for actual tokamak operations, a

Volume 2, Issue 1, 2021 
fixed alarm threshold is required, for offline studies such as those introduced in this work, the AUC of the ROC can effectively represent the general model performance.

\section{TRAINING AND PREDICTION RESULTS}

The data for this work come from the DIII-D tokamak located at General Atomics, San Diego, CA (http://www.ga.com/diii-d), and the EUROfusion JET tokamak located at the Culham Science Centre for Fusion Energy in the UK (https://www.euro-fusion.org/devices/jet/). The DIII-D data are sampled from shot numbers ranging from 125,500 to 168,555 , while the JET data are sampled from the carbon wall campaigns C23-C27b and the ITER-like wall campaigns C28-30. The different wall conditions in the two types of JET campaigns result in distinct plasma dynamics and disruption mechanisms. In this work, time-step size for the signal data is chosen to be $1 \mathrm{~ms}$. Using the same data selection and preprocessing procedure as described in the Methods section of Kates-Harbeck et al. (2019), we consider a shot to be valid within the database if and only if all of the relevant signals contain data for a period of time longer than the warning time. In order to assess model performance on DIII-D with a $1 \mathrm{~s}$ warning time, the DIII-D shots are required to have nonempty and nonflat data in all signals for at least $1 \mathrm{~s}$ in order to be considered "valid" and to thus be added to the FRNN training/validation/testing dataset. The numbers of shots in training, validation, and testing sets as listed in parentheses in Table 2.

In Table 2 we report the prediction results from four distinct FRNN architectures across several different experimental databases. Specifically, these four schemes are either LSTM-based or

TABLE 2: Prediction results of the tuned models on the test datasets, measured as AUCs at the warning time before a disruption

\begin{tabular}{|c|c|c|c|c|c|c|}
\hline & \multicolumn{4}{|c|}{ Single machine } & \multicolumn{2}{|c|}{ Cross Machine } \\
\hline $\begin{array}{l}\text { Training } \\
\text { (\#shots) }\end{array}$ & \multicolumn{3}{|c|}{ DIII-D (1702) } & JET-CW(1956) & DIII-D (2268) & JET-CW(1956) \\
\hline $\begin{array}{c}\text { Validation } \\
\text { (\#shots) }\end{array}$ & \multicolumn{3}{|c|}{ DIII-D (837) } & JET-CW(962) & DIII-D (1117) & JET-CW(962) \\
\hline $\begin{array}{c}\text { Testing } \\
\text { (\#shots) }\end{array}$ & \multicolumn{3}{|c|}{ DIII-D (846) } & JET-ILW(1133) & JET-ILW(1133) & DIII-D(846) \\
\hline $\begin{array}{c}\text { Warning } \\
\text { time }\end{array}$ & $30 \mathrm{~m}$ & $0.2 \mathrm{~s}$ & $1 \mathrm{~s}$ & $30 \mathrm{~ms}$ & $30 \mathrm{~ms}$ & $30 \mathrm{~ms}$ \\
\hline $\begin{array}{c}\text { FRNN } \\
\text { 0D-LSTM }\end{array}$ & 0.93 & 0.90 & 0.72 & 0.95 & 0.85 & 0.76 \\
\hline $\begin{array}{c}\text { FRNN } \\
\text { OD-TCN }\end{array}$ & 0.93 & 0.90 & 0.74 & 0.95 & 0.91 & 0.73 \\
\hline $\begin{array}{c}\text { FRNN } \\
\text { 1D-LSTM }\end{array}$ & 0.93 & 0.89 & 0.80 & - & 0.84 & - \\
\hline $\begin{array}{c}\text { FRNN } \\
\text { 1D-TCN }\end{array}$ & 0.93 & 0.91 & 0.79 & - & 0.86 & - \\
\hline
\end{tabular}


TCN-based models, each trained with and without the 1D profile information. We tuned hyperparameters for each architecture to select the best-performing model for each of the six different tasks. These six tasks are as follows: (i-iii) prediction of DIII-D disruptions with three different warning time cutoffs using models trained on separate DIII-D data; (iv-v) predictions for JET ITER-like wall shots disruptions with $30 \mathrm{~ms}$ warning time using models trained on JET carbon wall data, or on DIII-D data; and (vi) prediction for DIII-D disruptions with $30 \mathrm{~ms}$ warning time using models trained on JET carbon wall data. Since the JET carbon wall datasets did not include profile information, there are no FRNN 1D results for models trained or tested with these data.

To tune the hyperparameters of the neural network for each of the 20 entries corresponding to different predictive tasks in Table 2, we randomly selected 40 sets of hyperparameters chosen from a reasonable range of possible values, and then trained 40 models in parallel using the training and validation data. Main hyperparameters with their representative values for the TCN-based models are summarized in Table 3. In total, $800(20 \times 40)$ models were trained. After training all 40 models, we selected the best model based on its performance on the validation dataset. Finally, we examined the accuracy of each optimal model using the appropriate test dataset and summarized the test performance in Table 2 . The predictive performance is sensitive to the number of temporal and spatial convolutional filters, and the number of temporal convolutional blocks. When the size of the network is too small, it is not sufficient to represent the physical processes leading to disruptions. The predictions are only sensitive to the number of causal temporal convolutional layers when it is below 8 , indicating that the effective memory

TABLE 3: Hyperparameters to be tuned for the TCN based FRNN model, explanations of the hyperparameter symbol, and representative well-performing values

\begin{tabular}{|c|c|c|}
\hline Hyperparameter & Explanation & Representative value \\
\hline$\eta$ & Learning rate & $9.08 \mathrm{E}-5$ \\
$\mathrm{~N}_{\text {batch }}$ & Learning rate decay per epoch & 0.99 \\
$\mathrm{~T}_{\text {warning }}$ & Training batch size & 64 \\
& Warning time for target function, & 20 \\
Target & which becomes positive at $\mathrm{T}_{\text {warning }}$ & \\
& Type of target function & ttd (function linear \\
$\mathrm{NT}$ & Number of causal temporal & in time to disruption) \\
& convolutional layers & 8 \\
$\mathrm{NS}$ & Number of spatial convolutional layers & 2 \\
$\lambda$ & Weighting factor for positive examples & 16 \\
$\mathrm{~K}_{t}$ & Size of temporal convolutional filters & 11 \\
$\mathrm{~K}_{s}$ & Size of spatial convolutional filters & 7 \\
$\mathrm{~N}_{\mathrm{Tstack}}$ & Number of stacks of temporal & 2 \\
& convolutional blocks & 60 \\
$\mathrm{~N}_{t f}$ & Number of temporal convolutional filters & 20 \\
$\mathrm{~N}_{s f}$ & Number of spatial convolutional filters & 0.05 \\
Dropout & Dropout probability & \\
\hline
\end{tabular}

Volume 2, Issue 1, 2021 
of the plasma contributing to disruption is on the order of hundreds of milliseconds, consistent with the general plasma equilibrium evolution timescale.

Comparing results from the four schemes studied, the best-performing model for each task is highlighted in bold in Table 2. From this round of hyperparameter tuning run, for most of the tasks, the TCN-based model in FRNN performs equally well or better than the original LSTMbased model. As an example, the ROC curves for the best-performing models (based on TCN or LSTM) on the DIII-D test dataset with $0.2 \mathrm{~s}$ warning time and including 1D profiles are shown in Fig. 2. The two models perform equally well for the low false-positive rate regime, and the TCN-based model performs slightly better in the high true-positive rate regime. As the high true-positive rate regime is of greater importance to disruption prediction models for future machines that could not afford false-negative results, this result demonstrates an example where considering multiple deep-learning models can contribute to stronger predictive power for disruptions. Figure 3 shows an example DIII-D shot in the test dataset, and the output of these two models. While both models show some change in their disruption score shortly before the 1200 ms mark, only the TCN model shows a sufficient change to trigger a correct alarm. Near the end of the shot, the TCN model also outputs a higher disruption score, although this alarm is within the $30 \mathrm{~ms}$ warning time, and is considered "too late."

For the cross-machine prediction task where the model was trained only on DIII-D shots and is tasked to predict disruptions in the JET ITER-like wall shots, the TCN architecture offers significantly improved accuracy with less overfitting. The problem of overfitting is hard to overcome for this cross-machine predictive task due to drastically different physical parameters for DIII-D and JET plasmas. A comparison of the best TCN-based model and the best LSTMbased model performance on the cross-machine prediction task is shown in Fig. 4. Although the LSTM-based model demonstrates better performance on the training data from DIII-D plasmas, as shown in the left panel of Fig. 4, the TCN-based model achieves significantly better inference result for the JET data, as shown in the right panel of Fig. 4. The optimal alarm threshold estimated base on the DIII-D validation data also generalizes much better to the JET test data for the TCN-based model. This result indicates that the TCN-based model has learned deeper
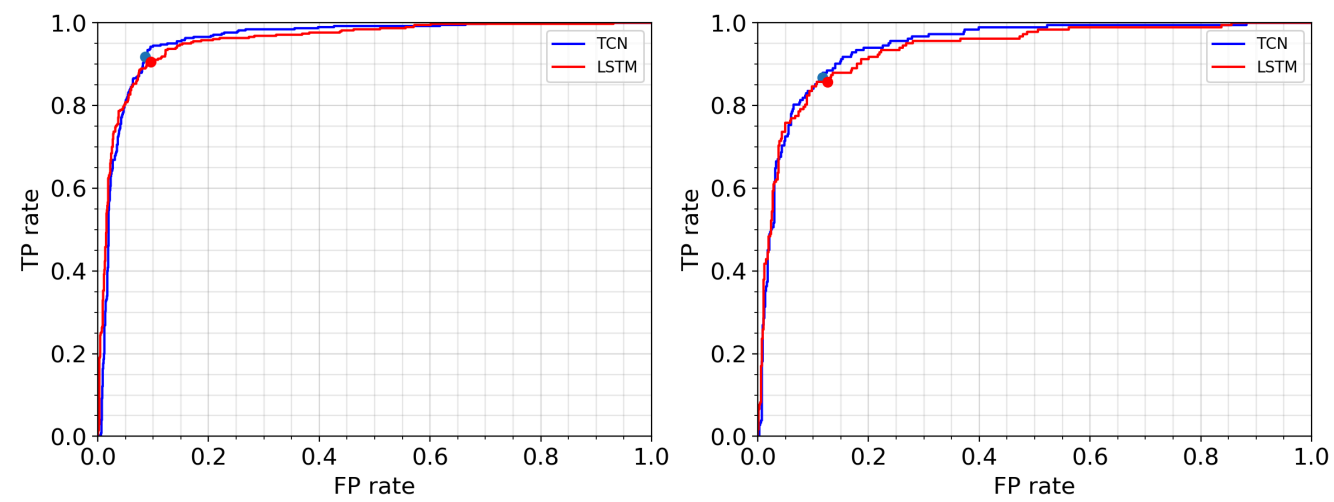

FIG. 2: Comparison of ROC curves on the DIII-D training (left panel) and test (right panel) dataset with $0.2 \mathrm{~s}$ warning time for the optimal FRNN 1D models, based on the TCN and LSTM architectures. The solid dots indicate model performance at the optimal alarm threshold determined on the validation set. Both models demonstrate good generalization of the optimal alarm threshold from DIII-D validation data to DIII-D test data. 


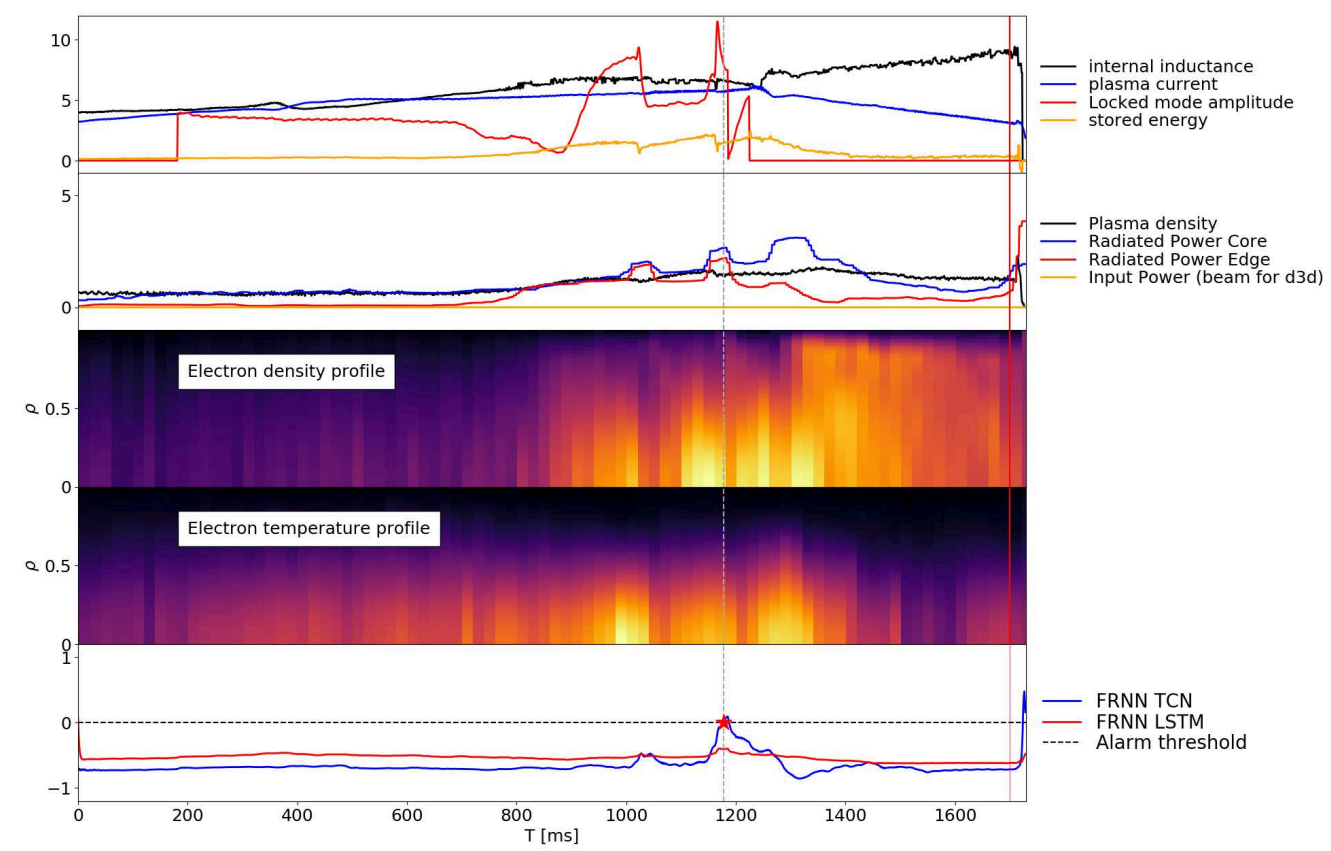

FIG. 3: Example prediction on DIII-D shot \#147206. The top two panels show eight representative 0D scalar signal channels (normalized by numerical scale on DIII-D as listed in Table 1), and the next two panels show two 1D profile signals channels as FRNN inputs. Vertical axis of the 1D profile signals are the normalized toroidal magnetic flux $\rho$. The last panel shows the disruption scores returned by FRNN using TCN and LSTM-based models. The FRNN TCN model raised positive disruption alarm before $1200 \mathrm{~ms}$ when reaching disruption alarm threshold indicated by the horizontal dashed line. The signals are plotted up to the time of disruption. The solid vertical red line shows the latest warning time $(30 \mathrm{~ms}$ before the disruption). It is important to highlight the finding here that while both models respond noticeably around the indicated disruption alarm time, only the TCN-based model correctly triggers the disruption alarm around $0.5 \mathrm{~s}$ before the actual disruption.

physics-based information, which is general for both plasma devices, and can thus achieve disruption predictions for plasma conditions that it has never seen during training.

Figure 5 shows the signals from an example JET shot in the top four panels, and outputs from the FRNN models trained on DIII-D database in the last panel, where only the TCN-based model is able to catch the continuously rising core radiation power as plotted in the second panel, and predict for the imminent disruption, even when the input power is decreased, as plotted in the second panel. On the contrary, the LSTM-based model output had a sharp rise in disruption score at around $12 \mathrm{~s}$, when the input power is suddenly increased.

Although in the two aforementioned tasks the TCN-based models outperform the LSTMbased models in FRNN, we highlight here that the LSTM-based modes can outperform the TCN-based models for other tasks, such as the prediction of disruptions in DIII-D plasmas when trained based on JET plasma signals, as shown in the last column in Table 2. It is therefore important to recognize that different types of deep-learning architectures can be suitable for different types of physics problems, and even different facets for the same problem, and the possible generally improved predictive powers if the ensemble model based on multiple diverse deep-learning architectures are considered, especially for cross-machine predictive tasks.

Volume 2, Issue 1, 2021 

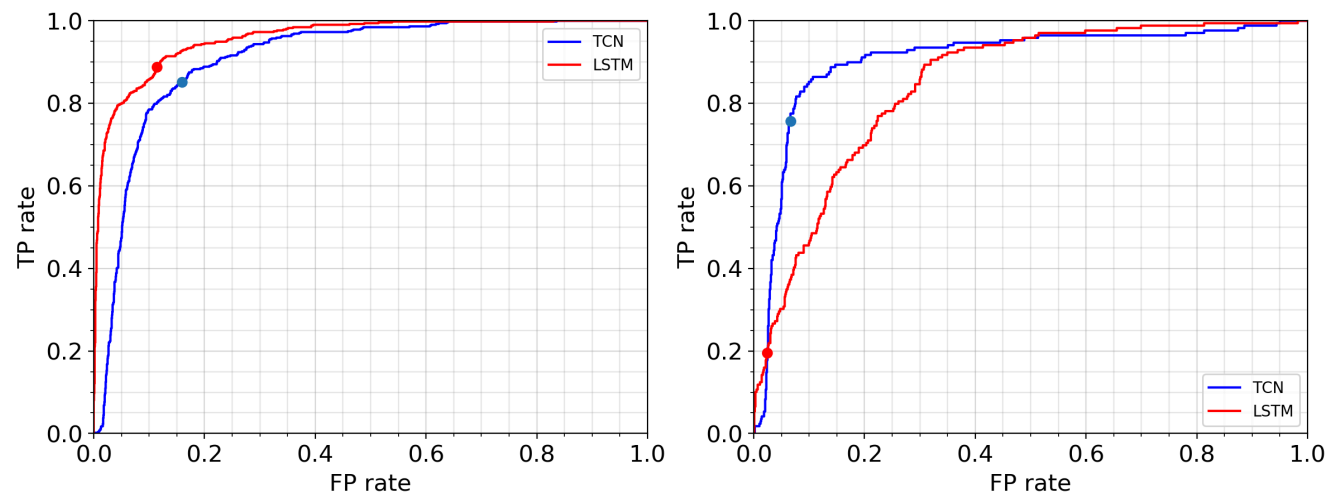

FIG. 4: Comparison of ROC curves on the DIII-D training (left panel) and JET test (right panel) dataset for the optimal FRNN 0D models, based on the TCN and LSTM architectures. The training and test score for the LSTM based model is 0.96 and 0.85 , respectively. For the TCN-based model, both training and test score is 0.91 , indicating much less overfitting. The solid dots indicate model performance at the optimal alarm threshold determined on the validation set. The generalization of the optimal alarm threshold from DIII-D validation set to JET test set is significantly better.

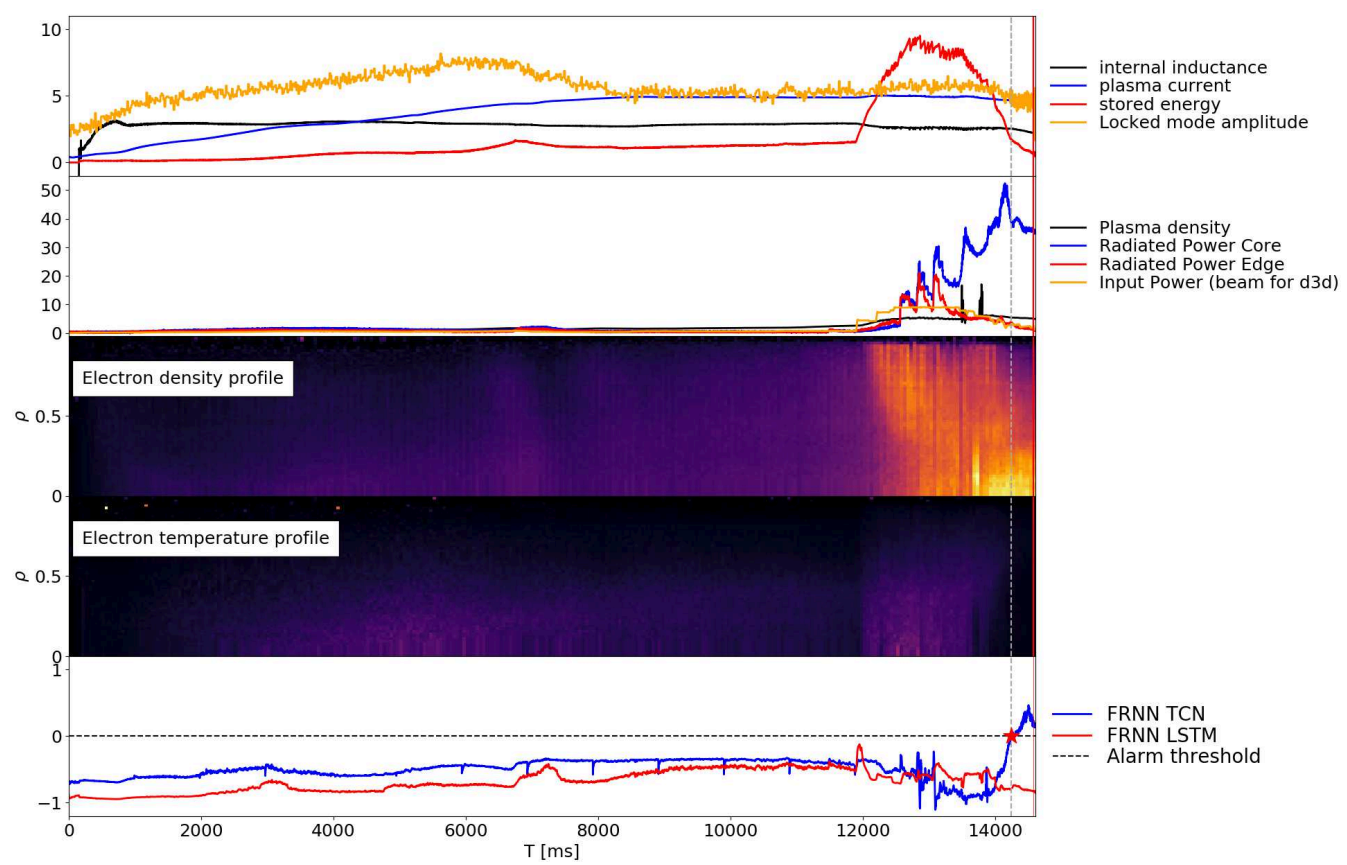

FIG. 5: Example prediction on JET shot \#83340. The top two panels show eight representative 0D scalar signal channels (normalized by numerical scale on JET as listed in Table 1), and the next two panels show two 1D profile signals channels as FRNN inputs. The last panel shows the disruption scores returned by FRNN using TCN and LSTM-based models. The signals are plotted up to the time of disruption. The dashed vertical line indicates the disruption alarm time, and the solid vertical line shows the latest warning time (30 ms before the disruption). 
In order to achieve an improved level of understanding of the physics and signal features underlying these disruption prediction results, we carried out a series of sensitivity studies. Specifically, we assessed the signal importance of all input signals for the single machine disruption prediction task on DIII-D using FRNN-1D. To assess the contribution from each of the 16 signals to the disruption scores, we trained 16 individual models each using one single signal at a time. In Fig. 6, test performances are compared for the FRNN-1D TCN-based (left panel) and the LSTM-based (right panel) models trained with the single labeled signal. As expected, for both architectural schemes, the models trained with single channels have significantly lower
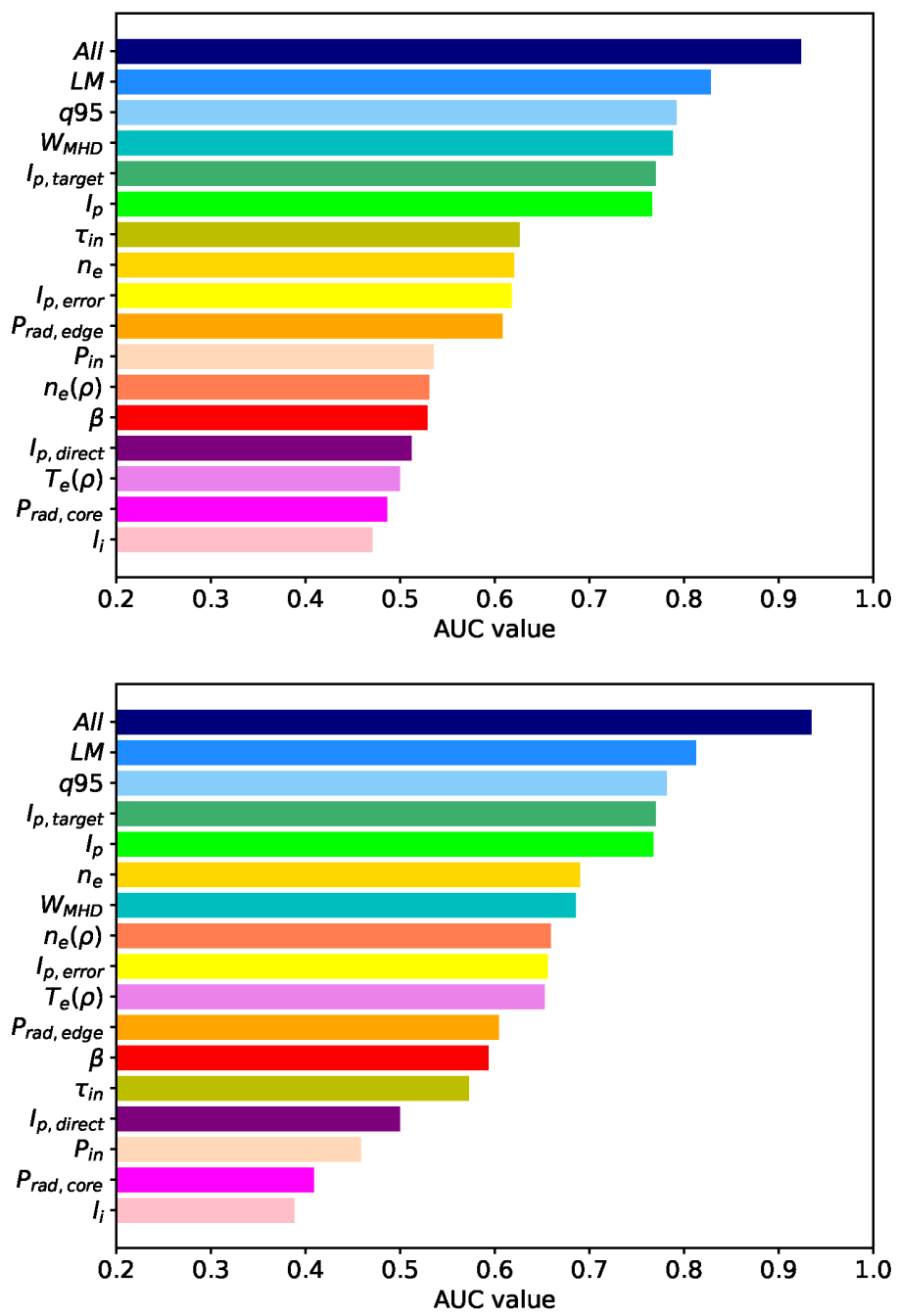

FIG. 6: Signal importance studies for models based on the TCN (left) and LSTM (right) architectures. Each bar represents the test set AUC values achieved by a model trained on the single labeled signal. The general trend of this sensitivity study is similar for the two architectures, showcasing the robustness of this method in estimating the relative importance of different physical signals.

Volume 2, Issue 1, 2021 
performance than the model trained with all 16 signals (represented by the deep blue bar). The signal importance as measured by model performance for each of the two models is affected by multiple factors, including initialization stochasticity and model hyperparameters. Therefore, some variation in signal importance values between the models is expected. However, it is important to note that there are clear qualitative trends that are consistent in both panels of Fig. 6. For example, the models trained on either the locked-mode amplitude or the tokamak safety factor value approaching the plasma periphery (Q95) signals outperform models trained on the rest of the signals, indicating that these two signals contain key disruption-related information.

In each panel in Fig. 6, all models are trained using the same set of hyperparameters as those used for the respective best model tuned with all signals (represented by the deep blue bar), and 34 models in total are trained for this analysis. In future investigations, more reliable estimates could be obtained by running hyperparameter tuning for each of these models, making it necessary to train thousands of models. Such a task would clearly require the associated engagement and effective utilization of modern supercomputers.

During real-time plasma experiments, due to unavailable diagnostic equipment, contaminated data collection process, or changing configuration of the experimental device and control system, some of the channels in the model inputs can be missing or containing high-amplitude noise. Therefore, we try to improve the robustness of our model performance with respect to input noise. There are many schemes to train noise-aware networks that can be stable against input noise (Seltzer and Wang, 2013; Zheng et al., 2016), here we use the simplest approach to perform noise-aware training by augmenting the training data with dropped out (set to zero) input channels to mimic unavailable experimental signals. The noise-aware models can significantly improve testing results when dropouts are added to the input data, at the cost of very small decrease in performance on the original full input. An example for DIII-D to JET cross-machine predictive task is shown in Table 4. When the channels in the input of the test dataset are dropped out with 0.1 probability (if the model is trained with 10 signals as input, then expectation of number of channels/signals that are set to zero is one) and 0.2 probability, the noise-aware model outperform the baseline model and is more robust on the perturbed data. The noise-aware training can help achieve predictive capabilities of the deep-learning-based models during real-time experiment in scenarios where certain diagnostics are unavailable or random data contamination happens.

TABLE 4: Prediction results on JET dataset from a model trained on the DIII-D datasets, measured as AUCs at $30 \mathrm{~ms}$ before a disruption

\begin{tabular}{|c|c|c|c|}
\hline Input channel dropout probability & 0 & 0.1 & 0.2 \\
\hline Baseline model & $\mathbf{0 . 9 0 2}$ & 0.847 & 0.798 \\
Noise-aware model & 0.900 & $\mathbf{0 . 8 7 5}$ & $\mathbf{0 . 8 3 9}$ \\
\hline
\end{tabular}

Noise-aware models achieve significantly more robust prediction results on inputs contaminated with dropped-out channels. The models are trained on 11 input signals, among which 2 channels are 1D profiles and 9 channels are 0D scalar signals. In the first column where input channel dropout probability is zero, the models are tested on the original full input data. In the second and third column the input channels in the test database are set to zero with probability 0.1 and 0.2 . The noise-aware model is trained with corresponding input dropout probability. In the first column the performance of the noise-aware model trained with 0.1 input dropout probability is reported. 


\section{COMPUTATIONAL PERFORMANCE EVALUATION}

As demonstrated in this paper, the training and tuning of deep-learning computational software like FRNN requires modern supercomputing power that must be utilized with excellent efficiency. Here, we demonstrate that with the new TCN architecture, FRNN exhibits even better computational performance. Specifically, Fig. 7 shows performance comparisons for the best TCN-based and the best LSTM-based models, when both are trained on the DIII-D database with $30 \mathrm{~ms}$ warning time. For this task, the key finding is that the TCN architecture enabled FRNN to reduce training time by about a factor of 2 .

As shown in Fig. 8, FRNN exhibits impressive strong scaling on the Oak Ridge Leadership Computing Facility (OLCF) "Summit" — currently the top-rated supercomputer worldwide (https://www.top500.org/list/2019/06/). Specifically, the training time for this TCN-based model scales almost ideally with the number of GPUs used. In this scaling study, we used a much larger database and a different set of model hyperparameters than that used in Fig. 7, to avoid

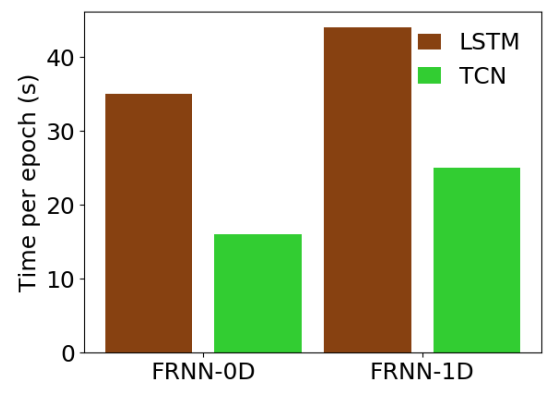

FIG. 7: Time per epoch (i.e., the time required to complete one pass over the entire training dataset) during training using 4 Tesla V100s for FRNN-0D and FRNN-1D, for the LSTM and TCN architectures, respectively. Lower values correspond to better computational performance. The four models studied here correspond to the best models from the studies of DIII-D single machine disruption prediction with $30 \mathrm{~ms}$ warning time (see first column in Table 2).

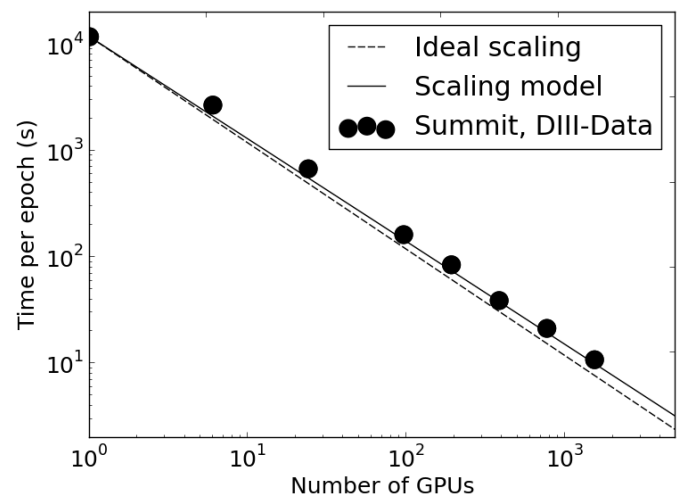

FIG. 8: Strong scaling of FRNN with the new TCN-based model carried out on the Oak Ridge Leadership Computing Facility (OLCF) Summit system-currently top-rated supercomputer worldwide. The time required to complete one epoch during training on Summit (black data points) agrees well with the scaling model. The original strong scaling of FRNN using the LSTM-based model and the associated derivation of the scaling model can be found in Kates-Harbeck et al. (2019).

Volume 2, Issue 1, 2021 
message passing interface (MPI) operation problems when the training time becomes too small for a large number of GPUs. This important finding motivates the future development of modern deep-learning software such as FRNN in various aspects-including performance optimization via hyperparameter tuning, consideration of more complicated and deeper architectures, and the addition of higher-dimensional input data sources with higher resolution.

\section{SUMMARY AND FUTURE WORK}

In this paper, we have introduced and implemented a new architectural scheme based on dilated temporal convolutions within the exemplar magnetic fusion plasma disruption prediction software FRNN. Compared with the previously published models based on the LSTM architecture, FRNN models constructed with the temporal convolutional neural network (TCN) architecture exhibit at least equivalent and demonstrably superior computational performance and predictive power for disruption forecasting across various experimental databases from the DIII-D and JET tokamaks. The TCN architecture has also been applied in other disruption studies recently, using input from the Electron Cyclotron Emission imaging (ECEi) diagnostic data on DIII-D (Churchill et al., 2020).

In the present paper, we have developed a general deep-learning capability to train multiple models with distinct architectures within a single software suite that serves to promote adaptability to different temporal and spatial learning tasks and also to enable ensemble schemes for highly accurate prediction. Various machine-learning-based algorithms targeting different learning and prediction tasks have been independently validated for modern tokamaks such as DIII-D (Rea et al., 2019) and JET (Rattà et al., 2010; Vega et al., 2014; Dormido-Canto et al., 2013; Murari et al., 2013; Ferreira et al., 2019). The capability of utilizing effective ensemble models in real-time plasma control systems is an important task for successful operation of future machines. To contribute to this effort, we plan to implement additional deep-learning-based architectures, including those based on attention-based models, such as the "Transformer" (Vaswani et al., 2017), into the FRNN framework, as well as utilizing more high-dimensional experimental data. The goal is to develop FRNN into a flexible and adaptable software platform for the prediction and analysis of complex plasma dynamics, including early disruption prediction as well as other important physics phenomena. Beyond plasma device performance predictions, this platform also has potential for wide applications to time series prediction problems in other research areas, such as magnetosphere substorm onset predictions and weather forecasts.

\section{ACKNOWLEDGMENTS}

Research for this paper was carried out at the Princeton Plasma Physics Laboratory by the Department of Energy (DOE) contract DE-AC02-09CH11466. The authors thank Professor Zhihong Lin of UC Irvine for useful discussions and important support of this work associated with the DOE SciDAC ISEP Center which he leads. We have benefited from the HPC resources of the Oak Ridge Leadership Computing Facility at the Oak Ridge National Laboratory (DOE contract No. DE-AC05-00OR22725) and the National Energy Research Scientific Computing Center (DOE contract No. DE-AC02-05CH11231. This work is also supported under contract No. DE-AC02-06CH11357 associated with the Argonne Leadership Computing Facility (ALCF) Aurora Early Science Program project at the Argonne National Laboratory. The simulations presented in this article were performed partly on computational resources featuring the "Traverse" 
cluster managed and supported by Princeton University's Research Computing Center, a consortium of groups including the Princeton Institute for Computational Science and Engineering (PICSciE) and the Office of Information Technology (OIT). We also express our gratitude to the EUROfusion Joint European Torus (JET) and their management as well as to General Atomics (GA) and its DIII-D tokamak project for access to the same fusion databases which were previously provided for the Nature (April, 2019) publication. In addition, we extend special thanks to Dr. Nik Logan of PPPL for his careful reading and associated helpful suggestions that improved the clarity of this manuscript. This material is based upon work supported by the US DOE, Office of Science, Office of Fusion Energy Sciences, using the DIII-D National Fusion Facility, a DOE Office of Science user facility, under award DE-FC02-04ER54698.

Disclaimer: This report was prepared as an account of work sponsored by an agency of the United States Government. Neither the United States Government nor any agency thereof, nor any of their employees, makes any warranty, express or implied, or assumes any legal liability or responsibility for the accuracy, completeness, or usefulness of any information, apparatus, product, or process disclosed, or represents that its use would not infringe privately owned rights. Reference herein to any specific commercial product, process, or service by trade name, trademark, manufacturer, or otherwise does not necessarily constitute or imply its endorsement, recommendation, or favoring by the United States Government or any agency thereof. The views and opinions of authors expressed herein do not necessarily state or reflect those of the United States Government or any agency thereof.

\section{REFERENCES}

Bai, S., Kolter, Z., and Koltun, V., An Empirical Evaluation of Generic Convolutional and Recurrent Networks for Sequence Modeling, accessed Jan. 31, 2021, from https://arxiv.org/abs/1803.01271, 2018.

Bengio, Y., Simard, P., and Frasconi, P., Learning Long-Term Dependencies with Gradient Descent Is Difficult, IEEE Trans. Neural Networks, vol. 5, no. 2, pp. 157-166, 1994.

Churchill, R.M., Tobias, B., Zhu, Y., and DIII-D Team, Deep Convolutional Neural Networks for MultiScale Time-Series Classification and Application to Tokamak Disruption Prediction Using Raw, High Temporal Resolution Diagnostic Data, Phys. Plasmas, vol. 27, no. 6, p. 062510, 2020.

de Vries, P.C., Pautasso, G., Humphreys, D., Lehnen, M., Maruyama, S., Snipes, J.A., Vergara, A., and Zabeo, L., Requirements for Triggering the ITER Disruption Mitigation System, Fusion Sci. Technol., vol. 69, pp. 471-484, 2019.

Dormido-Canto, S., Vega, J., Ramírez, J.M., Murari, A., Moreno, R., López, J.M., Pereira, A., and JETEFDA Contributors, Development of an Efficient Real-Time Disruption Predictor from Scratch on JET and Implications for ITER, Nucl. Fusion, vol. 53, no. 11, p. 113001, 2013.

EUROfusion Consortium Research Institutions, JET: EUROfusion's Flagship Device, accessed January 31, 2021, https://www.euro-fusion.org/devices/jet/, 2014.

Ferreira, D.R., Carvalho, P.J., and Fernandes, H., Deep Learning for Plasma Tomography and Disruption Prediction from Bolometer Data, IEEE Trans. Plasma Sci., vol. 48, pp. 36-45, 2019.

General Atomics, DIII-D, accessed January 31, 2021, from http://www.ga.com/diii-d, 2021.

Gerhardt, S.P., Darrow, D.S., Bell, R.E., LeBlanc, B.P., Menard, J.E., Mueller, D., Roquemore, A.L., Sabbagh, S.A., and Yuh, H., Detection of Disruptions in the High- $\beta$ Spherical Torus NSTX, Nucl. Fusion, vol. 53, no. 6, p. 063021, 2013.

Glasser, A.S. and Kolemen, E., A Robust Solution for the Resistive MHD Toroidal $\Delta$ Matrix in near RealTime, Phys. Plasmas, vol. 25, no. 8, p. 082502, 2018.

Volume 2, Issue 1, 2021 
Hollmann, E.M., et al., Status of Research toward the ITER Disruption Mitigation System, Phys. Plasmas, vol. 22, no. 021802, 2015.

Kates-Harbeck, J., Svyatkovskiy, A., and Tang, W., Predicting Disruptive Instabilities in Controlled Fusion Plasmas through Deep Learning, Nature, vol. 568, pp. 526-531, 2019

Liu, D., Zhang, W., McClenaghan, J., Wang, J., and Lin, Z., Verification of Gyrokinetic Particle Simulation of Current-Driven Instability in Fusion Plasmas. II. Resistive Tearing Mode, Phys. Plasmas, vol. 21, no. 12, p. 122520, 2014.

López, J.M., Vega, J., Alves, D., Dormido-Canto, S., Murari, A., Ramírez, J.M., Felton, R., Ruiz, M., de Arcas, G., and JET EFDA Contributors, Implementation of the Disruption Predictor APODIS in JET Real Time Network Using the MARTe Framework, 2012 18th IEEE-NPSS Real Time Conf., Berkeley, CA, pp. 1-4, 2012.

Matthews, G.F., et al., JET ITER-Like Wall-Overview and Experimental Programme, Phys. Scr., no. T145, p. 014001, 2011.

Murari, A., et al., Adaptive Predictors Based on Probabilistic SVM for Real Time Disruption Mitigation on JET, Nucl. Fusion, vol. 58, no. 5, p. 056002, 2018.

Oord, A., Dieleman, S., Zen, H., Simonyan, K., Vinyals, O., Graves, A., Kalchbrenner, N., Senior, A., and Kavukcuoglu, K., Wavenet, A Generative Model for Raw Audio, accessed Jan 31, 2021, from https://arxiv.org/abs/1609.03499, 2016.

Pautasso, G., et al., On-Line Prediction and Mitigation of Disruptions in ASDEX Upgrade, Nucl. Fusion, vol. 42, no. 1, p. 100, 2002.

Rattá, G.A., Vega, J., Murari, A., Vagliasindi, G., Johnson, M.F., de Vries, P.C., and JET EFDA Contributors, An Advanced Disruption Predictor for JET Tested in a Simulated Real-Time Environment, Nucl. Fusion, vol. 50, no. 2, p. 025005, 2010.

Rea, C., Montes, K.J., Erickson, K.G., Granetz, R.S., and Tinguely, R.A., A Real-Time Machine LearningBased Disruption Predictor in DIII-D, Nucl. Fusion, vol. 59, no. 9, p. 096016, 2019.

Reichstein, M., Camps-Valls, G., Stevens, B., Jung, M., Denzler, J., Carvalhais, N., and Prabhat, Deep Learning and Process Understanding for Data-Driven Earth System Science, Nature, vol. 566, pp. 195204, 2019.

Schmidt, J., Marques, M.R.G., Botti, S., and Marques, M.A.L., Recent Advances and Applications of Machine Learning in Solid-State Materials Science, NPJ Comput. Mater, vol. 5, no. 83, pp. 1-36, 2019.

Schuller, F., Disruptions in Tokamaks, Plasma Phys. Controlled Fusion, vol. 37, no. 11A, p. A135, 1995.

Seltzer, M.L., Yu, D., and Wang, Y., An Investigation of Deep Neural Networks for Noise Robust Speech Recognition, 2013 IEEE Int. Conf. on Acoustics, Speech and Signal Processing, Vancouver, BC, pp. 7398-7402, 2013.

Strait, E.J., et al., Progress in Disruption Prevention for ITER, Nucl. Fusion, vol. 59, no. 11, p. 112012, 2019.

Top500.org, List of Top 500, November 2020, accessed January 31, 2021, https://www.top500.org/lists/ top500/2020/11/,2020.

Vaswani, A., Shazeer, N., Parmar, N., Uszkoreit, J., Jones, L., Gomez, A.N., Kaiser, L., and Polosukhin, I., Attention Is All You Need, Adv. Neural Inf. Process. Syst., pp. 6000-6010, 2017.

Vega, J., Murari, A., Dormido-Canto, S., Moreno, R., Pereira, A., Acero, A., and JET-EFDA Contributors, Adaptive High Learning Rate Probabilistic Disruption Predictors from Scratch for the Next Generation of Tokamaks, Nucl. Fusion, vol. 54, no. 12, p. 123001, 2014.

Webb, S., Deep Learning for Biology, Nature, vol. 554, pp. 555-557, 2018.

Zheng, S., Song, Y., Leung, T., and Goodfellow, I., Improving the Robustness of Deep Neural Networks via Stability Training, Proc. of the IEEE Conf. on Computer Vision and Pattern Recognition (CVPR), pp. 4480-4488, 2016. 